\title{
Realization of an Antireflection Nanopore Array for Black Si Solar Energy Utilization
}

\author{
Ze-ping LI \\ School of Electronic Information and Engineering \\ Hubei University of Science and Technology \\ Xianning, 437005, PR China \\ School of Optical and Electronic Information, \\ Huazhong University of Science and Technology \\ Wuhan, 430074, PR China \\ e-mail: lzpind@hust.edu.cn

\section{Gang XIONG} \\ School of Electronic Information and Engineering \\ Hubei University of Science and Technology \\ Xianning, 437005, PR China
}

\author{
Li-hong MEI* \\ School of Electronic Information and Engineering \\ Hubei University of Science and Technology \\ Xianning, 437005, PR China \\ e-mail: 215839665@qq.com \\ www.hbust.com.cn
}

\begin{abstract}
We demonstrate that an antireflection nanopore arrays were fabricated for black Si solar energy utilization using the highly ordered anodic aluminum oxide (AAO) masks. We have studied the optical characteristics of antireflection nanopore arrays and polished $\mathrm{Si}$. It is found that the reflectivity of the surface of as-prepared $\mathrm{Si}$ nanopore arrays decreases from around $22 \%$ to less than $1 \%$ in the wavelength region from 300 to $1100 \mathrm{~nm}$. The achieved excellent antireflection property compared with polished $\mathrm{Si}$ can increase solar cell efficiency, our inexpensive $\mathrm{Si}$ production method would lower the cost of solar-grade Si materials.
\end{abstract}

Keywords- Antireflection; Black Si; Nanopore arrays; AAO

\section{INTRODUCTION}

Solar energy has been considered having the potential for future worldwide energy demand and reduces the dependence on fossil fuel sources, because of its inexhaustive, sustainable and environmental friendliness [1,2]. In the last 50 years, commercial silicon photovoltaics have been developed to convert sunlight into electricity at efficiencies about 20\% [3]. Due to high refractive index of $\sim 4$ in the visible wavelength range, silicon reflects most of the visible light incident onto the surface. This high surface reflectivity is undesirable for applications of silicon in solar energy. The traditional technique to lower the reflection of silicon surface is to deposit a single or multilayer dielectric film based on the quarter wavelength design that reduces the reflection only for a narrowband of wavelengths and does not exihibit wide angle-of-view [4]. To resolve the problem, a nanostructured $\mathrm{Si}$ known as black $\mathrm{Si}$ is adopted $[5,6]$ due to its unique optical and electrical characteristics [7,8]. Different from traditional antireflection coatings, black $\mathrm{Si}$ has a broadband antireflection at a far wider acceptance angle than single or multilayer antireflection films [9]. High efficiencies have been recently demonstrated by black $\mathrm{Si}$ solar cells, which provided improved antireflection and light trapping functionality compared with the traditional $\mathrm{Si}$ [10]. The resulting lower reflection and improved light trapping can improve short circuit currents [11], with the addition of higher fill factors and voltages than traditional cells, which increase solar cell efficiencies and enable the use of low grade Si raw materials, thus reducing the manufacturing cost [12].

In this work, we demonstrate that an antireflection nanopore arrays are fabricated for black Si solar energy utilization using the highly ordered AAO masks. We have studied the optical characteristics of antireflection nanopore arrays and polished $\mathrm{Si}$. It is found that $\mathrm{Si}$ nanopore arrays that can dramatically reduce optical reflection compared with polished Si.

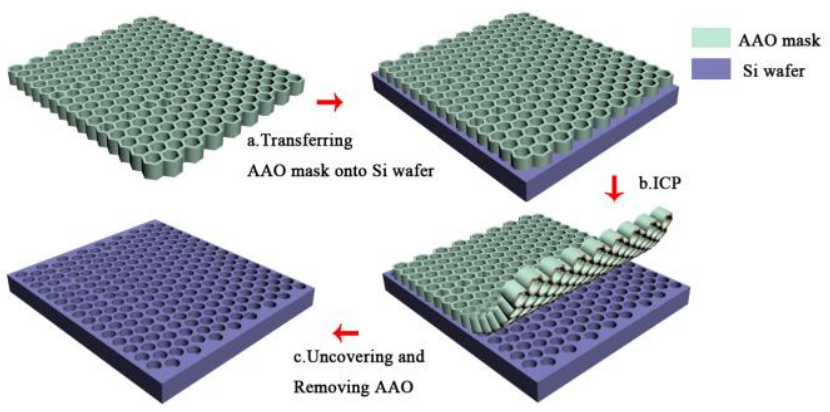

Fig. 1. Preparation of Si nanopore arrays; (a) The AAO mask was transferred onto $\mathrm{Si}$ wafer, (b) The Si was etched by inductive coupled plasma, (c) The AAO was uncovered and removed from Si wafer.

\section{EXPERIMENTAL DETAILS}

\section{A. Mask Preparation}

The AAO mask was prepared by the two-step anodization process as our previous work [13]. In brief, annealing, cleaning and electrochemical polishing were 
taken on the 0.2-mm-thick aluminum foil ( $99.999 \%$ in purity) prior to the anodization process. The first anodization was carried out in $1 \%$ phosphoric acid and $0.01 \mathrm{M}$ aluminum oxalate hydrate for $4 \mathrm{~h}$ at the temperature of $1{ }^{\circ} \mathrm{C}$ and voltage of $195 \mathrm{~V}$, followed by the $1.8 \%$ chromic acid and $6 \%$ phosphoric acid aqueous solution for $8 \mathrm{~h}$ to remove the irregular oxide layer. The second anodization was carried for $2 \mathrm{~h}$ under the same conditions. Subsequently, the unreacted aluminum were dissolved in a saturated aqueous solution of copper chloride before pore-widening and pore-opening process finished in $5 \%$ phosphoric acid solution for $50 \mathrm{~min}$ at $50^{\circ} \mathrm{C}$.

\section{B. Mask Transferring}

The prepared AAO mask was transferred onto a 2 inch ntype (boron-doped) (100) Si wafer with a resistance ranging from $0.001-0.015 \Omega \cdot \mathrm{cm}$ and a thickness of $500 \pm 25 \mu \mathrm{m}$, which was cleaned by Piranha solution $(\mathrm{H} 2 \mathrm{SO} 4: \mathrm{H} 2 \mathrm{O} 2$, v/v = 3/1) for $5 \mathrm{~min}$, followed by being ultrasonically cleaned in acetone, methanol, and deionized (DI) water, and then dried with nitrogen. The transferring process was carried out in DI water for keeping the AAO mask from twisting, folding and cracking. In the process, the AAO mask successfully was transferred onto Si wafer. After the DI water on Si wafer was dried on the hotplate, the commercial tape was used to fix the AAO mask on Si wafer (figure 1(a)).

\section{Fabrication of Nanopore Arrays}

The nanopore arrays were prepared on the $\mathrm{Si}$ wafer through the transferred AAO mask which acted as the etching mask (figure 1(b)). After transferring the AAO mask onto the wafer, the AAO mask was fixed on the wafer using double-sided tape in order to avoid moving of AAO mask. Subsequently, the exposed surface of Si wafer inside the pore channel was etched (etching rate of $5 \mathrm{~nm} / \mathrm{s}$ ) by C4F8 and SF6 plasma etching at 20 mTorr and $900 \mathrm{~W}$ for 100s. The flow rates of C4F8 and SF6 are 15 and $12 \mathrm{sccm}$, respectively. Finally, the AAO mask was removed with the commercial tape (figure 1(c)).

\section{RESULTS AND DISCUSSION}

In order to show the whole structure of the AAO mask/Si nanopore arrays, parts of the AAO mask were removed intentionally, which resulted in parts of retained mask as shown in figure 2(a). Figure 2(a) demonstrates highly ordered Si nanopore arrays with diameter of 400nm below highly ordered AAO mask.

Utilizing standard $\mathrm{Si}$ nanofabrication technology, large scale highly orderly Si nanopore arrays can be fabricated on Si wafer. Sub-ten-nanometer pattern generation commonly utilizes electron beam lithography, which is time-consuming and expensive $[14,15]$. Another popular Si nanofabrication technology is standard RIE, which is aided by advanced lithography techniques, [16,17] such as photolithography, nanosphere lithography, and nanoimprint lithography.
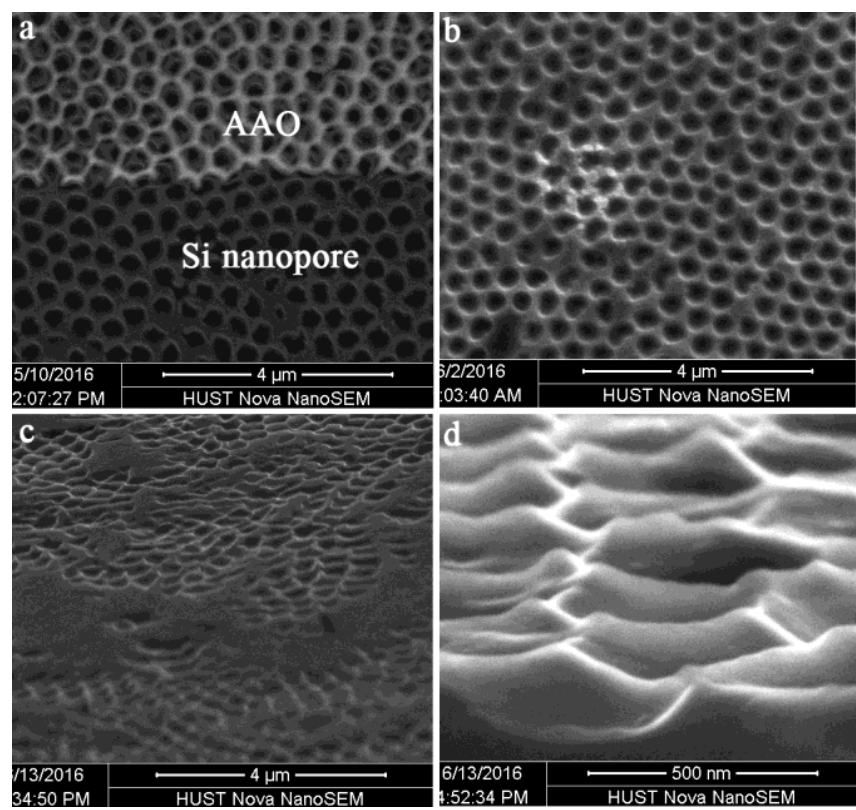

Fig. 2. SEM images of (a) the plane view of Si nanopore arrays with AAO mask, (b) the plane view of large scale Si nanopore arrays, (c) the oblique view of large scale Si nanopore arrays, (d) the oblique view of Si nanopore arrays in high resolution.

In our method, $\mathrm{Si}$ nanopore arrays were directly fabricated by inductive coupled plasma using AAO mask as the etching mask. Large scale highly ordered $\mathrm{Si}$ nanopore arrays are shown on figure 2(b) after AAO mask is removed. Obviously, Si nanopore arrays in figure 2(b) demonstrate hexagonal packed distribution with the same structure distribution of AAO mask. In the process of inductive coupled plasma, the plasma passed through the AAO pore channel and etched the exposed surface of Si inside the pores. The oblique view image of large scale Si nanopore arrays is shown in figure 2(c), which contains the contaminant of tape from SEM measurements. The oblique view image of Si nanopore arrays in high resolution in figure 2(d) shows hemispherical structure of $\mathrm{Si}$ nanopore, which dues to anisotropic etching in process of inductive coupled plasma. In our top-down process, $\mathrm{Si}$ nanopore arrays inherit the electrical characteristics of the mother $\mathrm{Si}$ wafer and do not need further doping processing for conductivity [18], which is beneficial to their application in black Si solar energy.

The optical absorption properties of Si nanopore arrays are important in conversion efficiency of solar energy. To analyze the reflectivity performance of the $\mathrm{Si}$ nanopore arrays, the reflectivity was measured by a UV-vis spectrometer (JASCO V-670) equipped with an integral sphere. Figure 3 shows the wavelength dependence of the reflectivity (at an incidence of $8^{\circ}$ ). The black curve at the top is the reflectivity of polished silicon, and red curve is reflectivity of the as-prepared $\mathrm{Si}$ nanopore arrays. In the wavelength region from 300 to $1100 \mathrm{~nm}$, the reflectivity of the surface of as-prepared Si nanopore arrays decreases from around $22 \%$ to less than $1 \%$. However, the polished $\mathrm{Si}$ sample shows a reflectance of $43 \%$ at $300 \mathrm{~nm}$ and decreases to an almost constant value of around $36 \%$ for wavelengths 
ranging between 350 and $1100 \mathrm{~nm}$. The Si nanopore arrays sample shows a small reflectance value at almost whole range of wavelengths measured and comes near to the zero value at $550 \mathrm{~nm}$. Consequently, the $\mathrm{Si}$ nanopore arrays drastically suppress light reflection over a wide spectral bandwidth. This remarkably low reflectance of the $\mathrm{Si}$ nanopore arrays is attributed to several advantages: (1) the large surface area; (2) the sub-wavelength light-trapping effects of Si nanopore arrays; and (3) the collective light scattering interactions among $\mathrm{Si}$ nanopore arrays, which trap light and make it travels many turns over distances much longer than the pore thickness. This excellent antireflection properties contributes to enhancing light-trapping effects of Si nanopore arrays, which results in excellent absorption of sunlight above the Si bandgap.

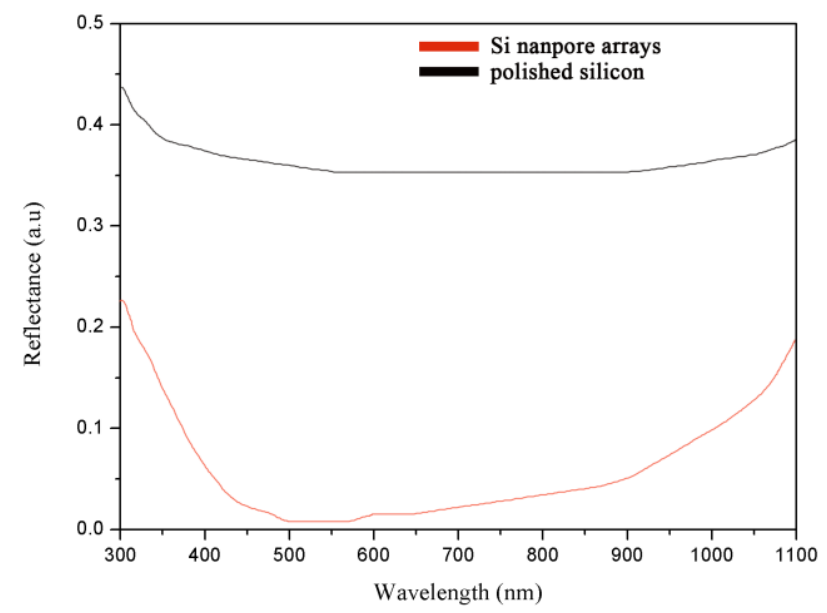

Fig. 3. Reflectance measurements of Si nanopore arrays and polished Si.

\section{CONCLUSIONS}

In summary, the as-prepared $\mathrm{Si}$ nanopore arrays possess distinctive optical properties owing to their high surface areas and diameters close to visible wavelengths, the $\mathrm{Si}$ nanopore arrays using our top-down method inherit the electrical characteristics of the mother $\mathrm{Si}$ wafer and do not need further doping processing for conductivity, which are beneficial to black Si solar energy application. The presented process will be suitable for the large-scale production of ordered nano-semiconductor arrays, which would lower the cost of solar-grade Simaterials via inexpensive Si production methods

\section{ACKNOWLEDGMENT}

This work is supported by grant ky14049 of Hubei University of Science and Technology Foundation.

Work partially supported by grant 2015AA043302 of National High-tech R\&D Program (863 Program) of China; grant 61474048 of National Natural Science Foundation of China; grant XYJ2014000198 of Science and Technology Support Program declaration of Hubei Province of China; grant HUST2015049 of The central university basic scientific research business expenses.

\section{REFERENCES}

[1] X.X. Liu, X.Y. Hu, H. Miao, G.W. Zhang, J.L. Mu, T.X. Han and D.K. Zhang, SOL ENERGY, 134, 45-51 (2016).

[2] L.T. Lin, X.M. Wu, T. W, D.J. Chen, C. D, J.X. Meng and L.W. Cao, J ALLOY COMPD, 673, 411-419 (2016).

[3] N.S. Lewis and D.G. Nocera, Proc. Natl. Acad. Sci. U.S.A. 104, 20142-20152 (2007).

[4] J. Zhao and M.A. Green, IEEE Trans. Electron Devices, 38, 1925-34 (1991).

[5] X. Liu, P.R. Coxon, M. Peters, B. Hoex, J.M. Cole and D.J. Fray, Energy Environ. Sci. 7, 3223-63 (2014).

[6] U.A. Yusufoglu, T.M. Pletzer, L.J. Koduvelikulathu, C. Comparotto, R. Kopecek and H. Kurz, IEEE J. Photovolt. 5, 320-8 (2015).

[7] J. Zhu, Z. Yu, G. F. Burkhard, C. Hsu, S. T. Connor, Y. Xu, Q. Wang, M. McGehee, S. Fan, and Y. Cui, Nano Lett. 9, 279 (2009).

[8] R. A. Street, P. Qi, R. Lujan and W. S. Wong, Appl. Phys. Lett. 93, 163109 (2008).

[9] L.L. Ma, Y.C. Zhou, N. Jiang, X. Lu, J. Shao, W. Lu, J. Ge, X.M. Ding and X.Y. Hou, Appl. Phys. Lett. 88, 171907 (2006).

[10] F. Toor, J.B. Miller, L.M. Davidson, W. Duan, P.M. Jura, J. Yim, J. Forziati and M.R. Black, Nanoscale, 8, 15448-66 (2016).

[11] F. Toor, H.M. Branz, M.R. Page, K.M. Jones and H.C. Yuan, Appl. Phys. Lett. 99, 103501 (2011).

[12] E. Cabrera, S. Olibet, D. Rudolph, E. Wefringhaus, R. Kopecek, D. Reinke and G. Schubert, IEEE J. Photovolt. 3, 102-7 (2013).

[13] T.Y. Sun, W.N. Zhao, X.H. Wu, S.S. Liu, Z.Z. Ma, J. Peng, J. He , H.F. Xu, S.Y. Liu and Z.M. Xu, J LIGHTWAVE TECHNOL, 32, 326-332 (2014).

[14] R. Juhasz, N. Elfstrom and J. Linnros, Nano Lett. 5, 275 (2005).

[15] H.D. Tong, S. Chen, W.G. van der Wiel, E.T. Carlen and A. van den Berg, Nano Lett. 9, 1015 (2009).

[16] C.M. Hsu, S.T. Connor, M.X. Tang and Y.Cui, Appl. Phys. Lett. 93, 133109 (2008).

[17] E. Garnett and P.D. Yang, Nano Lett. 10, 1082 (2010).

[18] K.Q. Peng and S.T. Lee, Adv. Mater. 23, 198-215 (2011) 